\title{
Risk Factors Associated with Coronary Artery Calcification Should Be Examined before Kidney Transplantation
}

\author{
Sanja Simic-Ogrizovic, ${ }^{1,2}$ Natasa Bogavac-Stanojevic, ${ }^{3}$ Maja Vuckovic, ${ }^{4}$ \\ Violeta Dopsaj, ${ }^{3}$ Voja Giga, ${ }^{5}$ Milica Kravljaca, ${ }^{1}$ Milan Stosovic ${ }^{1}$ and Visnja Lezaic ${ }^{1,2}$ \\ ${ }^{1}$ Nephrology Clinic, Clinical Centre of Serbia, Belgrade, Serbia \\ ${ }^{2}$ School of Medicine, Belgrade University, Belgrade, Serbia \\ ${ }^{3}$ Institute of Medical Biochemistry, Faculty of Pharmacy, Belgrade, Serbia \\ ${ }^{4}$ Institute of Radiology, Clinical Centre of Serbia, Belgrade, Serbia \\ ${ }^{5}$ Cardiology Clinic, Clinical Centre of Serbia, Belgrade, Serbia
}

\begin{abstract}
The best treatment for end stage renal disease (ESRD) patients is kidney transplantation, but the renal transplant recipients still have a higher incidence of cardiovascular events compared with general population. Cardiovascular risk factors were imposed long before ESRD, as the majority of patients starting dialysis or kidney transplantation already have signs of advanced atherosclerosis. Artery calcification is an organized, regulated process similar to bone formation. Coronary artery calcification (CAC) is found frequently in advanced atherosclerotic lesions and could be a useful marker of them. We evaluated the prevalence of CAC in 49 stable renal transplant recipients and in 48 age- and gender-matched patients with chronic kidney disease (CKD) in stages 2-5 not requiring dialysis to assess risk factors associated with CAC. Computed tomography was used for CAC detection and quantification (CAC score). The prevalence of CAC was $43.8 \%$ in transplant recipients and $16.7 \%$ in CKD patients $(p<0.001)$. Transplant recipients with CAC were significantly older and had longer duration of CKD and/or dialysis than recipients without CAC. In contrast, the serum levels of fetuin A (an inhibitor of vascular calcification) and albumin were significantly lower in CKD patients with CAC than those without CAC. During the observation period (30 months), 30 patients, including 23 CKD patients, began dialysis, and 4 transplant recipients and 2 CKD patients died. Independent predictors of mortality were age, serum amyloid A and the CAC score. In conclusion, the examination and prevention of risk factors associated with atherosclerosis should be started at the beginning of renal failure.
\end{abstract}

Keywords: chronic kidney disease; coronary artery calcification; fetuin A; inflammation; renal transplant recipient Tohoku J. Exp. Med., 2012, 226 (2), 137-144. (C) 2012 Tohoku University Medical Press

The standard treatment for patients with end stage renal disease (ESRD) is kidney transplantation as it considerably prolongs life mainly by impeding the progression of cardiovascular disorders by improving renal function (Meier-Kriesche et al. 2004). On the other hand, although the transplanted kidney provides cardiac and renal benefits, kidney function still remains poorer than that of healthy subjects. Renal transplant recipients have a much higher incidence of cardiac death and non-fatal cardiovascular events compared with the general population (Liefeldt and Budde 2010).

During the last 15 years after the introduction of more sophisticated imaging techniques including multi-slice or electron bean computed tomography, coronary artery calcification (CAC) has not ceased to be a topic of interest for many researchers in the field of nephrology. Vascular calcification is more frequent (2-5 fold) and more severe in uremic patients than in age- and gender-matched individuals in the general population (Braun et al. 1996). Goodman et al. (2000) indicated that a high prevalence of CAC in young patients is especially marked in those who had been on dialysis for more than 10 years. In addition, both intimal and medial artery calcification occur in uremic patients and represent significant predictors for $\mathrm{CV}$ morbidity and mortality in this population (Schwarz et al. 2000).

During the last decade, significant attention has been focused towards factors implicated in the pathophysiology of CAC in end stage renal disease. Some of the factors include poor calcium and phosphate homeostasis, intact parathyroid hormone (iPTH) concentration, oxidative stress, inflammation and levels of vascular and soft tissue calcification inhibitors, together with age, dialysis vintage, dyslipidemia, diabetes, some medication and genetic factors (Martola et al. 2005). Currently accepted opinions propose that in the uremic milieu, vascular calcifications may result from both an imbalance in divalent ions and a deficit of

Received November 9, 2011; revision accepted for publication January 17, 2012. doi: 10.1620/tjem.226.137

Correspondence: Sanja Simic-Ogrizovic, Nephrology Clinic, Clinical Centre of Serbia, Pasterova 2, Belgrade 11000, Serbia.

e-mail: ssogrizovic@gmail.com 
inhibitory proteins, eventually leading to phenotypic change and calcium deposition (Ketteler et al. 2005). It could be expected that, after successful kidney transplantation and definite improvement of the uremic milieu, the imbalance between promoters and inhibitors of vascular calcification would be lessened (Mazzaferro et al. 2007). However, the prevalence of renal transplant patients with CAC still remains very high.

We evaluated the prevalence of CAC in our stable renal transplant recipients as well as age- and gendermatched patients with chronic kidney disease (CKD) in stages 2-5 not requiring dialysis, and assessed possible demographic and laboratory risk factors associated with CAC in these two different groups. We thus identified significant predictors of patient mortality after a 30 -month follow-up period.

\section{Patients and Methods}

Patients

This prospective (30 month duration) observational study involved 49 stable renal transplant (Tx) recipients, at least 6 months after transplantation and $48 \mathrm{CKD}$ patients in stages 2-5 not requiring dialysis. CKD patients were selected from the population regularly monitored at the Nephrology Clinic in order to obtain a group of similar age and gender as the Tx patients. Diabetics and patients with acute inflammatory diseases were excluded.

The characteristics of all study participants are shown in Table 1. Thirty-six patients had received a kidney from a related living donor and thirteen from a deceased donor. These patients had previous transplantation duration of $9.56 \pm 5.27$ years. Any history of hypertension, ischemic vascular disease (myocardial infarction, angina pectoris, cerebral stroke), heart failure and smoking habits was noted at the study entry interview.

The original kidney diseases in our patients were: chronic glomerulonephritis ( $n=29$ in Tx and $n=16$ in CKD patients), chronic pyelonephritis and congenital urinary tract anomalies $(n=5$ in Tx and $n=4$ in CKD patients), nephrosclerosis ( $n=3$ in Tx and $n=14$ in CKD patients), polycystic kidney disease ( $n=2$ in Tx and $n=6$ in CKD patients), other ( $n=3$ in Tx and $n=8$ in CKD patients) and unknown ( $n=7$ in Tx and $n=0$ in CKD patients). The immunosuppressive protocol for Tx patients consisted of calcineurin inhibitors (cyclosporine or tacrolimus), mycophenolate acids or azathioprine and prednisolone. CKD patients with some kind of glomerulonephritis as the original disease received corticosteroids or other immunosuppressive therapy according to protocols.

Clinical and immunological observations, as well as laboratory parameters and coronary calcium score using computed tomography were determined on the same day.

All patients gave informed consent prior to their enrolment in the study, which was planned according to the ethical guidelines following the Declaration of Helsinki. The institutional review committee approved our study protocol thereby following local biomedical research regulations.

\section{Laboratory methods}

A fasting blood sample was drawn from each patient once in order to measure the following parameters: complete blood count and serum concentrations of uric acid, creatinine (s-creatinine), albumin, pre-albumin, transferrin, calcium, phosphate, iPTH, cholesterol, triglycerides, high sensitive CRP (hs-CRP), serum amyloid A (SAA), fetuin A, total homocysteine (tHcy) and interleukin-6 (IL-6). Hematological profiles were determined using an LH 750 hematology analyser (Beckman Coulter Inc., California, USA). Creatinine, uric acid and albumin were analyzed employing routine methods (Olympus System Reagents using an AU 2700 Olympus analyser, Hamburg, Germany). iPTH was determined by ELISA-PTH, (CIS bio international, GIF-sur-Yvette Cedex, France). hs-CRP, SAA, prealbumin and transferrin were measured using immunonephelometric assays (Dade-Behring, BN II, Marburg, Germany). Serum IL-6 levels were determined with a highly sensitive colorimetric sandwich ELISA kit (Human IL-6 Quantikine HS ELISA kit; R\&D Systems, GmbH, Germany). Serum fetuin A was determined by ELISA (Epitope Diagnostics, Inc., San Diego, California, USA).

Serum tHcy concentration was measured by high-performance liquid chromatography (HPLC) after reduction of the disulfide-bonds by dithiothreitol (normal range: $10-15 \mu \mathrm{mol} / \mathrm{l}$ ).

Glomerular filtration rate (GFR) was estimated using the abbreviated Modification of Diet in Renal Disease (MDRD) formula study equation (Levey et al. 2006).

Arterial hypertension was diagnosed when the systolic blood pressure was $\geq 140 \mathrm{mmHg}$ and/or diastolic pressure was $\geq 90 \mathrm{mmHg}$, or if antihypertensive treatment was prescribed.

Body mass index (BMI) was calculated according to the formula: weight $(\mathrm{kg}) /$ height $^{2}\left(\mathrm{~m}^{2}\right)$.

\section{Coronary artery calcification (CAC) score}

CAC score was evaluated using multi-detector row spiral computed tomography (MSCT) (General Electric Medical System, USA) operating with the following parameters: 64 slices with 912 detectors, $0.625 \mathrm{~mm}$ distance between slices, $0.35 \mathrm{~s}$ rotation time and a tube current of $800 \mathrm{~mA}$ at $120 \mathrm{kV}$. Data were obtained during the diastolic phase of the heart cycle. CAC score calculations were based on formulae using measurements of total volume and area of calcified lesions, as well as mean and maximum density. Individual CAC scores were calculated for the left main coronary artery, descending branch of the left coronary artery, circumflex branch of the left coronary artery and right coronary artery. Scores then were summed to calculate the total coronary CAC score. The final score is expressed in modified Agatston units (Agatston et al. 1990).

To identify factors associated with CAC, renal Tx and CKD patients were separated into two sub-groups according to the presence or absence of significant CAC using a cut-off score of 100. This calcium score was chosen according to the latest guidelines from the American College of Cardiology and the American Society of Nuclear Cardiology endorsed by the American Heart Association (ACC/ASNC) (Greenland et al. 2007).

The database included demographic (age and gender), clinical (CKD, dialysis and Tx duration, BMI, hypertension), CAC score as well as hematological, biochemical and immunological variables.

\section{Statistics}

Differences in continuous variables between the groups were analysed by the Student's $t$-test for normally distributed variables. Values for S-creatinine, proteinuria, triglycerides, hs-CRP, IL-6 and SAA were log-transformed to achieve normality. Due to the fact that the CAC score distribution and disease duration were skewed after log-transformation, these variables were compared by the Mann- 
Whitney test. Adjusted mean levels were estimated by analysis of covariance (ANCOVA). Group differences for categorical variables were examined by the Chi-square test. Uni-variate associations were evaluated by Spearman's correlation analysis.

Clinical accuracy of the examined parameters was assessed using receiving operative characteristic (ROC) curve analysis. The area under the ROC curve (AUC) is considered to be a useful quantitative measure of accuracy. The ROC curve allows variable thresholds to be determined on a target sensitivity or specificity. We set the cut-off value at the point when discrimination between the cases with positive and negative diagnosis was optimal.

In order to analyse the death risk, uni-variate survival analysis with the Cox proportional hazard model was obtained. The primary dependent variable was the time to death measured in months. Variables that were potential predictors of death in uni-variate analy- sis were tested in the multi-variate Cox proportional hazard model.

All calculations were performed using MS Excel, EduStat 2.01 (2005, Alpha Omnia, Belgrade, Serbia) and MedCalc for Windows version Version 9.6.3 (Mariakerke, Belgium). Minimal statistical significance was set at $p<0.05$.

\section{Results}

Comparisons between renal Tx and CKD patients are presented in Table 1. Renal Tx patients had a significantly higher CAC score, CKD and/or dialysis duration and significantly higher inflammatory markers (hs-CRP, SAA and IL-6) along with lower serum fetuin A levels but better kidney function, higher tHcy concentrations and significantly lower serum phosphate levels and $\mathrm{Ca} \times \mathrm{P}$ compared with

Table 1. Demographic characteristics and laboratory data of renal Tx and CKD patients.

\begin{tabular}{|c|c|c|c|}
\hline & Renal Tx patients & CKD patients & $p$ \\
\hline$N$ & 49 & 48 & \\
\hline Age (years) & $41.98 \pm 10.58$ & $38.21 \pm 15.56$ & ns \\
\hline Gender $(\mathrm{M} / \mathrm{F})$ & $31 / 18$ & $25 / 23$ & ns \\
\hline Smoking history $(\%)$ & 20 & 26.5 & ns \\
\hline Hypertension (\%) & 58 & 51 & ns \\
\hline IVD $(\%)$ & 14.2 & 10.4 & ns \\
\hline $\mathrm{CKD}$ and/or D duration (months) $\dagger$ & $143(97-206)$ & $24(12-33)$ & $<0.001^{\mathrm{a}}$ \\
\hline Transplantation duration (months) & $114.28 \pm 63.28$ & / & / \\
\hline CAC score $\dagger$ & $51(0-461.25)$ & $0(0-7.5)$ & $0.002^{\mathrm{a}}$ \\
\hline BMI $\left(\mathrm{kg} / \mathrm{m}^{2}\right)$ & $24.94 \pm 3.56$ & $24.40 \pm 3.42$ & $\mathrm{~ns}$ \\
\hline S. creatinine $(\mu \mathrm{mol} / \mathrm{L}) *$ & $172.44(154.64-192.29)$ & $249.84(208.11-299.94)$ & $<0.001$ \\
\hline GFR $\left(\mathrm{ml} / \mathrm{min} / 1.73 \mathrm{~m}^{2}\right)$ & $39.60 \pm 15.04$ & $30.86 \pm 22.15$ & 0.026 \\
\hline Haemoglobin $(g / L)$ & $124.92 \pm 22.14$ & $128.34 \pm 22.31$ & ns \\
\hline Uric acid $(\mu \mathrm{mol} / \mathrm{L})$ & $412.02 \pm 91.3$ & $470.46 \pm 112.85$ & 0.006 \\
\hline Albumin $(\mathrm{g} / \mathrm{L})$ & $41.17 \pm 6.09$ & $42.20 \pm 3.57$ & ns \\
\hline Pre-albumin $(\mathrm{g} / \mathrm{L})$ & $0.32 \pm 0.11$ & $0.32 \pm 0.10$ & ns \\
\hline Transferrin $(\mathrm{g} / \mathrm{L})$ & $2.16 \pm 0.53$ & $2.16 \pm 0.50$ & ns \\
\hline Calcium (mmol/L) & $2.42 \pm 0.18$ & $2.34 \pm 0.20$ & 0.56 \\
\hline Phosphate (mmol/L) & $1.16 \pm 0.28$ & $1.48 \pm 0.41$ & $<0.001$ \\
\hline $\mathrm{Ca} \times \mathrm{P}\left(\mathrm{mmol}^{2} / \mathrm{L}^{2}\right)$ & $2.7 \pm 0.56$ & $3.44 \pm 0.86$ & $<0.001$ \\
\hline intact PTH $(\mathrm{pg} / \mathrm{mL}) *$ & $103.49(77.53-138.14)$ & $86.35(67.05-111.21)$ & 0.360 \\
\hline Cholesterol (mmol/L) & $5.8 \pm 1.11$ & $5.88 \pm 1.24$ & ns \\
\hline Triglycerides $(\mathrm{mmol} / \mathrm{L}) *$ & $1.89(1.66-2.17)$ & $1.81(1.55-2.12)$ & ns \\
\hline hs-CRP $(\mathrm{mg} / \mathrm{L}) *$ & $1.38(0.897-2.131)$ & $0.73(0.477-1.118)$ & 0.037 \\
\hline $\mathrm{SAA}(\mathrm{mg} / \mathrm{L}) *$ & $8.54(6.389-11.427)$ & $3.38(2.585-4.311)$ & $<0.001$ \\
\hline IL-6 $(\mathrm{pg} / \mathrm{mL}) *$ & $4.16(3.43-5.039)$ & $2.40(1.95-2.957)$ & $<0.001$ \\
\hline Homocysteine (umol/l) & $24.27 \pm 11.0$ & $19.91 \pm 6.71$ & 0.016 \\
\hline Fetuin A $(\mathrm{g} / \mathrm{L})$ & $0.397 \pm 0.133$ & $0.463 \pm 0.111$ & 0.014 \\
\hline
\end{tabular}

CKD, chronic kidney disease; D, dialysis; Tx, transplantation; CAC, coronary artery calcification; GFR, glomerular filtration rate; hs-CRP, high sensitive C-reactive protein; SAA, serum amyloid A; IL-6, interleukin 6.

Continuous variables are presented as mean \pm standard deviation and compared by Student $t$ test, whereas categorical variables are presented as relative frequencies and compared using the Chi-square test.

$\dagger$ Values for CKD and/or D duration and CAC score are presented as median and $25^{\text {th }}$ and $75^{\text {th }}$ percentile.

*Values for S-creatinine, intact PTH, triglycerides, hs-CRP, IL-6 and SAA are presented as geometrical mean and 95\% confidence intervals (CI). Logarithmic transformation of the values was performed before the analysis.

a variables are compared using the Mann-Whitney test. 


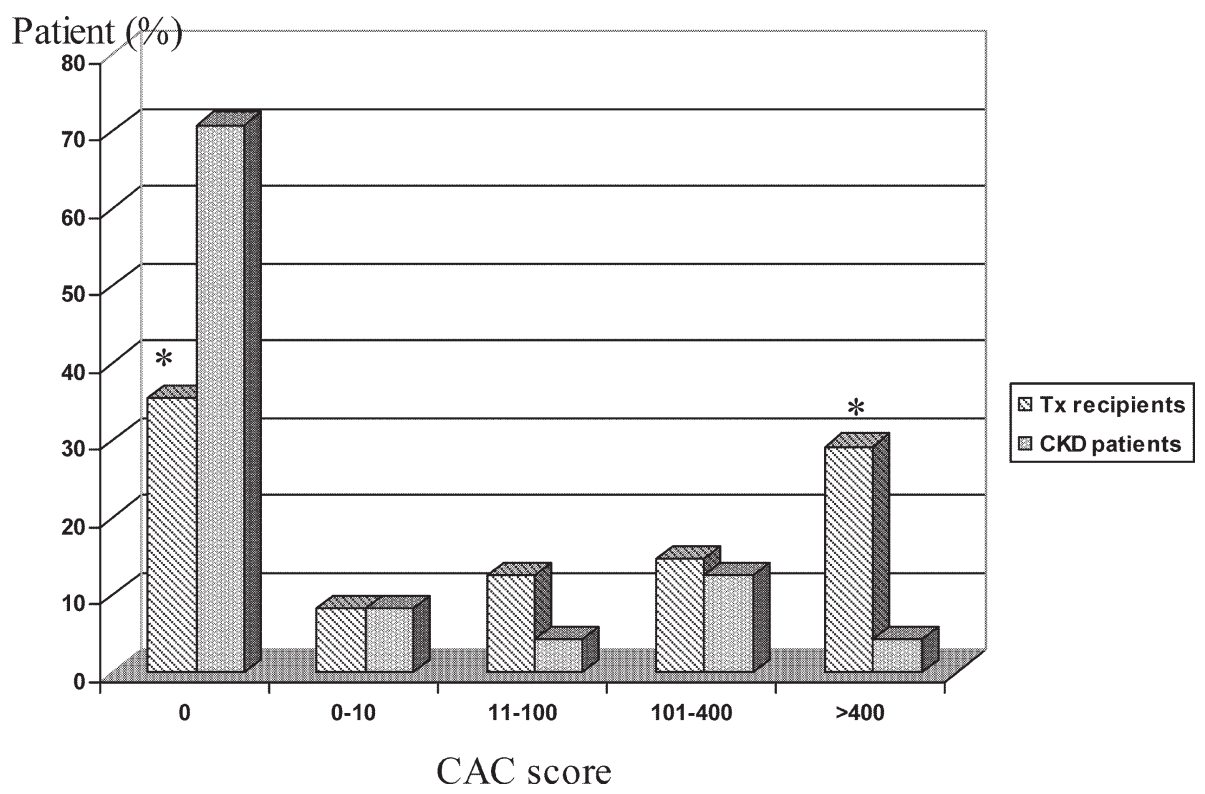

Fig. 1. CAC score in transplant recipients and $\mathrm{CKD}$ patients. Tx, renal transplant recipients $\mathrm{CKD}$, chronic kidney disease patients

\section{CKD patients.}

The prevalence of patients with a CAC score $>100$ was $31 \%$ for all the examined patients; it was $43.8 \%$ in the Tx group and $16.7 \%$ in the CKD patients $(p<0.001)$.

More than half the patients (54\%) had a CAC score of zero $(35.4 \%$ in the Tx recipients compared with $70.8 \%$ in CKD patients; $p<0.001$ ), while 7\% had CAC scores $1-10$ (8.3\% compared with $8.3 \% ; p=0.976), 8 \%$ values of $11-100(12.5 \%$ compared with $4.2 \% ; p=0.148), 14 \%$ CAC scores of $101-400(14.6 \%$ compared with $12.5 \%, p=0.796)$ and $17 \%$ of the patients had CAC scores $>400(29.2 \%$ compared with $4.2 \% ; p=0.001$ ) (Fig. 1).

For the Tx patients Spearman analysis revealed significant correlations only between CAC score and age $(r=$ $0.480, p=0.001)$, hypertension $(r=0.303, p=0.036)$ and uric acid $(r=0.321, p=0.026)$. In CKD patients the CAC score was positively associated with age $(r=0.693, p<$ $0.001)$, hypertension $(r=0.349, p=0.025)$, CKD duration $(r$ $=0.442, p=0.002)$ and albumin $(r=0.303, p=0.037)$ but negatively with serum fetuin A concentration $(r=-0.419$, $p=0.004$ ).

Table 2 shows the comparison between patients with a CAC score $\leq 100$ (without CAC) and with a CAC score > 100 (with CAC). Tx patients with CAC were significantly older and had longer duration of CKD and/or dialysis than Tx patients without CAC. In addition, Tx patients with CAC showed significantly increased serum uric acid, compared to those without CAC. The serum IL-6 level tended to be higher and the serum fetuin A level tended to be lower in renal Tx recipients with CAC, compared to those without CAC, although the differences were not statistically significant.

CKD patients with CAC were older, had longer CKD duration and higher tHcy concentrations but lower serum levels of albumin, pre-albumin and fetuin A (Table 2).

To explore whether the association between albumin, pre-albumin, tHcy and fetuin A and CAC score were confounded by other factors, the concentrations of these parameters in CKD patients with and without CAC were compared after adjusting for age and duration of disease. Serum albumin concentrations $(F=4.551, p=0.039)$ and fetuin A $(F=15.897, p=0.001)$ were significantly lower in CKD patients with CAC than the corresponding values in CKD patients without CAC. However, no significant differences were found for adjusted means of the other parameters between CKD patients with and without CAC. In Tx patients adjusted uric acid for age and CKD duration were not significantly different between Tx patients with and without CAC.

The AUC was calculated to assess the accuracy of the examined parameters in screening a CAC score $>100$ in CKD patients. The significant ROC curves with specificities and sensitivities for optimal cut-off values are presented in Table 3. Only fetuin A and albumin had acceptable discriminative ability for detecting CAC presence (Swets 1988). The ROC curves for hsCRP, IL-6 and pre-albumin indicated moderate accuracy for discrimination between patients with and without CAC. No other parameters indicated significant discriminative ability for detecting CAC. Although the sensitivity of fetuin A, albumin, pre-albumin and hsCRP were equal $(100 \%)$, their specificities ranged from $72.2 \%$ for fetuin A to $61.1 \%$ for pre-albumin. In $\mathrm{Tx}$ recipients no parameter had significant discriminative ability for detecting CAC.

During the observational period (30 months), 30 patients began dialysis ( $7 \mathrm{Tx}$ recipients and $23 \mathrm{CKD}$ 
Table 2. Demographic characteristics and laboratory data in renal Tx and CKD patients without CAC and with CAC (CAC score $>$ $100)$.

\begin{tabular}{|c|c|c|c|c|c|c|}
\hline & \multicolumn{3}{|c|}{ Renal Tx patients } & \multicolumn{3}{|c|}{ CKD patients } \\
\hline & $\begin{array}{l}\text { Without CAC } \\
\quad(n=28)\end{array}$ & $\begin{array}{l}\text { With CAC } \\
(n=21)\end{array}$ & $P$ & $\begin{array}{l}\text { Without CAC } \\
\quad(n=40)\end{array}$ & With CAC $(n=8)$ & $P$ \\
\hline Age (years) & $36.2 \pm 8.96$ & $46.67 \pm 9,370$ & $<0.002$ & $31.8 \pm 9.72$ & $62.6 \pm 6.52$ & $<0.001$ \\
\hline CKD and/or D duration (months) $\dagger$ & $\begin{array}{c}132 \\
(62-154)\end{array}$ & $\begin{array}{c}161 \\
(113-228)\end{array}$ & $<0.001^{\mathrm{a}}$ & $\begin{array}{c}12 \\
(6-24)\end{array}$ & $\begin{array}{c}42 \\
(42-105)\end{array}$ & $<0.001^{\mathrm{a}}$ \\
\hline $\operatorname{BMI}\left(\mathrm{kg} / \mathrm{m}^{2}\right)$ & $24.72 \pm 3.68$ & $24.87 \pm 3.33$ & ns & $24.0 \pm 3.05$ & $25.9 \pm 4.48$ & ns \\
\hline S. creatinine $(\mu \mathrm{mol} / \mathrm{L})^{*}$ & $\begin{array}{c}164.93 \\
(141.38-192.40)\end{array}$ & $\begin{array}{c}181.22 \\
(154.18-213.01)\end{array}$ & ns & $\begin{array}{c}248.47 \\
(202.16-305.39)\end{array}$ & $\begin{array}{c}255.09 \\
(157.93-412.03)\end{array}$ & ns \\
\hline $\operatorname{GFR}\left(\mathrm{ml} / \mathrm{min} / 1.73 \mathrm{~m}^{2}\right)$ & $41.54 \pm 15.07$ & $37.13 \pm 14.42$ & ns & $31.92 \pm 23.07$ & $26.87 \pm 18.78$ & ns \\
\hline Haemoglobin $(\mathrm{g} / \mathrm{L})$ & $121.67 \pm 20.56$ & $126.37 \pm 23.18$ & ns & $124.53 \pm 22.91$ & $127.45 \pm 21.72$ & ns \\
\hline Uric acid $(\mu \mathrm{mol} / \mathrm{L})$ & $385.48 \pm 90.79$ & $438.00 \pm 87.54$ & 0.048 & $485.84 \pm 109.5$ & $452.0 \pm 111.43$ & ns \\
\hline Albumin $(\mathrm{g} / \mathrm{L})$ & $42.17 \pm 4.59$ & $42.28 \pm 21.81$ & ns & $45.8 \pm 2.86$ & $39.95 \pm 6.08$ & 0.005 \\
\hline Pre-albumin $(\mathrm{g} / \mathrm{L})$ & $0.32 \pm 0.13$ & $0.33 \pm 0.11$ & ns & $0.35 \pm 0.01$ & $0.23 \pm 0.12$ & 0.002 \\
\hline Transferrin $(\mathrm{g} / \mathrm{L})$ & $2.19 \pm 0.64$ & $2.13 \pm 0.475$ & ns & $2.16 \pm 0.55$ & $2.19 \pm 0.27$ & ns \\
\hline Calcium (mmol/L) & $2.42 \pm 0.13$ & $2.42 \pm 0.23$ & ns & $2.34 \pm 0.217$ & $2.36 \pm 0.17$ & ns \\
\hline Phosphate $(\mathrm{mmol} / \mathrm{L})$ & $1.14 \pm 0.27$ & $1.19 \pm 0.31$ & ns & $1.51 \pm 0.433$ & $1.39 \pm 0.37$ & ns \\
\hline $\mathrm{Ca} \times \mathrm{P}\left(\mathrm{mmol}^{2} / \mathrm{L}^{2}\right)$ & $2.71 \pm 0.62$ & $2.84 \pm 0.53$ & ns & $3.50 \pm 0.61$ & $3.24 \pm 0.69$ & ns \\
\hline inatct PTH $(\mathrm{pg} / \mathrm{mL})$ * & $\begin{array}{c}97.82 \\
(62.73-152.54)\end{array}$ & $\begin{array}{c}112.53 \\
(74.62-169.72)\end{array}$ & ns & $\begin{array}{c}77.41 \\
(59.04-101.5)\end{array}$ & $\begin{array}{c}130.09 \\
(64.40-262.77)\end{array}$ & ns \\
\hline Cholesterol (mmol/L) & $5.49 \pm 1.17$ & $6.07 \pm 1.04$ & ns & $5.78 \pm 1.26$ & $6.26 \pm 1.14$ & ns \\
\hline Triglycerides $(\mathrm{mmol} / \mathrm{L})^{*}$ & $\begin{array}{c}1.68 \\
(1.41-2.00)\end{array}$ & $\begin{array}{c}1.97 \\
(1.66-2.34)\end{array}$ & ns & $\begin{array}{c}1.92 \\
(1.61-2.29)\end{array}$ & $\begin{array}{c}1.45 \\
(1.01-2.10)\end{array}$ & ns \\
\hline $\mathrm{CRP}(\mathrm{mg} / \mathrm{L}) *$ & $\begin{array}{c}1.56 \\
(0.70-3.51)\end{array}$ & $\begin{array}{c}1.30 \\
(0.75-2.26)\end{array}$ & ns & $\begin{array}{c}0.61 \\
(0.37-0.99)\end{array}$ & $\begin{array}{c}1.74 \\
(0.94-3.21)\end{array}$ & ns \\
\hline $\mathrm{SAA}(\mathrm{mg} / \mathrm{L})^{*}$ & $\begin{array}{c}8.54 \\
(5.87-12.43)\end{array}$ & $\begin{array}{c}8.55 \\
(45.40-13.52)\end{array}$ & ns & $\begin{array}{c}3.33 \\
(2.43-4.55)\end{array}$ & $\begin{array}{c}3.38 \\
(2.26-5.05)\end{array}$ & ns \\
\hline IL-6 $(\mathrm{pg} / \mathrm{mL})^{*}$ & $\begin{array}{c}3.65 \\
(2.31-5.67)\end{array}$ & $\begin{array}{c}4.63 \\
(3.47-6.16)\end{array}$ & ns & $\begin{array}{c}2.16 \\
(1.41-2.74)\end{array}$ & $\begin{array}{c}3.51 \\
(2.28-5.38)\end{array}$ & ns \\
\hline Homocysteine (umol/1) & $22.03 \pm 6.48$ & $25.94 \pm 12.57$ & ns & $18.5 \pm 5.76$ & $24.15 \pm 7.96$ & 0.037 \\
\hline Fetuin A $(\mathrm{g} / \mathrm{L})$ & $0.411 \pm 0.148$ & $0.385 \pm 0.120$ & ns & $0.494 \pm 0.101$ & $0.354 \pm 0.068$ & $<0.001$ \\
\hline
\end{tabular}

CKD, chronic kidney disease; D, dialysis; Tx, transplantation; CAC, coronary artery calcification; GFR, glomerular filtration rate; hs-CRP, high sensitive C-reactive protein; SAA, serum amyloid A; IL-6, interleukin 6.

Continuous variables are presented as mean \pm standard deviation and compared by Student $t$ test, whereas categorical variables are presented as relative frequencies and compared using the Chi-square test.

$\dagger$ Values for CKD and/or D duration are presented as median and $25^{\text {th }}$ and $75^{\text {th }}$ percentile.

* Values for S-creatinin, intact PTH, triglycerides, hs-CRP, IL-6 and SAA are presented as geometrical mean and 95\% confidence intervals (CI). Logarithmic transformation of the values was performed before the analysis.

a variables are compared using the Mann-Whitney test

Table 3. The results of ROC analysis for discriminating CKD patients without and with CAC.

\begin{tabular}{lcccrrr}
\hline & AUC & Confidence interval & Std. error & Cut-off & Specificity & Sensitivity \\
\hline Fetuin A (g/L) & 0.878 & $0.777-0.978$ & 0.051 & 4.37 & 1.00 & 0.722 \\
Albumin (g/L) & 0.826 & $0.710-0.942$ & 0.059 & 42.00 & 1.00 & 0.632 \\
hs-CRP (mg/L) & 0.763 & $0.631-0.895$ & 0.067 & 0.57 & 1.00 & 0.684 \\
Pre-albumin (g/L) & 0.750 & $0.608-0.892$ & 0.072 & 0.32 & 1.00 & 0.611 \\
IL-6 (pg/mL) & 0.744 & $0.572-0.917$ & 0.088 & 4.10 & 0.600 & 0.889 \\
\hline
\end{tabular}

AUC statistically different from 0.5

hs-CRP, high sensitive C-reactive protein; IL-6, interleukin 6; AUC, area under the ROC curve. 
Table 4. Predictors of mortality using the multi-variate Cox proportional hazard model.

\begin{tabular}{lccc}
\hline \multicolumn{1}{c}{ Variable } & $\beta$ & Hazard ratio $(\mathrm{CI})$ & $p$ \\
\hline Age & .229 & $1.257(1.006-1.482)$ & 0.007 \\
CAC score & .001 & $1.001(1.000-1.002)$ & 0.020 \\
SAA & .074 & $1.077(1.020-1.137)$ & 0.008 \\
\hline
\end{tabular}

CAC, coronary artery calcification; SAA, serum amyloid A.

patients), one Tx recipient suffered a myocardial infarction, and six patients died (2 CKD patients due to cardiovascular events and 4 renal Tx patients - 2 due to cardiovascular events, 1 from a malignancy and 1 from a severe pulmonary infection).

Predictors of mortality using the uni-variate Cox proportional hazard model in all examined patients were: age $(p=0.003), \mathrm{CKD} /$ dialysis duration $(p=0.004), \mathrm{CAC}$ score $(p=0.001)$, tHcy $(p=0.014)$, SAA $(p=0.001)$, IL-6 $(p=$ $0.005)$ and fetuin A $(p<0.001)$. All significant predictors of mortality were subjected to a forward stepwise selection process. In multi-variate analysis age, SAA and CAC score were found to be independent predictors of patient death (Table 4).

\section{Discussion}

In the present study the prevalence of CAC in $\mathrm{Tx}$ patients was $43.8 \%$ and was significantly higher than in CKD patients (16.7\%). Rosas et al. (2005) found CAC in $65 \%$ of Tx recipients but $66 \%$ of the examined patients were diabetics. In other studies with significantly fewer diabetics a similar prevalence of CAC was found in $\mathrm{Tx}$ patients (Bargnoux et al. 2009; Seyahi et al. 2011). In the large Dallas Heart Study among 2,660 participants aged 43.9 years, Kramer et al. (2005) observed a $25 \%$ prevalence of a CAC score of $>10$ and $15 \%$ of CAC scores $>100$ in 28 non-diabetic stage 3-5 CKD patients, but in 13 diabetics stage 3-5 CKD patients the prevalence of a CAC score $>10$ was $85 \%$. In a multi-ethnic study of atherosclerosis Kestenbaum et al. (2009) found a prevalence of CAC at $66 \%$ in much older patients (68.7 years) with CKD stage 3.

Moe et al. (2004) and Mazzafero et al. (2007) compared mean CAC score, potential promoters and inhibitors for CAC and outcome in Tx recipients with values for dialysis patients. The Tx patients had CAC scores of lower severity, no net change in CAC with time (Moe et al. 2004), less inflammation (Mazzaferro et al. 2007), higher levels of calcification inhibitors (Mazzaferro et al. 2007) and a better survival rate than dialysis patients (Moe et al. 2004). However, we evaluated Tx recipients with age and gender matched stage 2-5 CKD patients not requiring dialysis. Although the Tx patients had better kidney function and lower serum uric acid levels, they had significant higher CAC scores and positive acute phase proteins (hs-CRP, SAA and IL-6) along with lower serum fetuin A levels than CKD patients. After successful kidney transplantation inflammation markers significantly decreased because of faster cytokine clearance and an improved uremic milieu but some degree of inflammation still persisted or was elevated despite immunosuppressive therapy. Vascular inflammation is an important factor in the development of atherosclerosis as illustrated by the increased number of posttransplant cardiovascular events with elevated levels of hsCRP in transplant recipients (Bakri et al. 2004). In our study Tx patients had greater CAC scores as a surrogate of atherosclerosis than CKD patients and this could be the reason for greater inflammation in these patients despite better kidney function.

In addition, our renal $\mathrm{Tx}$ recipients had significantly lower serum phosphate levels and $\mathrm{Ca} \times \mathrm{P}$ than $\mathrm{CKD}$ patients with no difference in iPTH levels. It is well known that after successful kidney transplantation significant changes occur in divalent ion concentrations. In particular, serum phosphate may reach normal or even low levels (Mazzaferro et al. 2009).

As in most other studies examining dialysis (Kraśniak et al. 2007) or Tx patients (Rosas et al. 2005; Mazzaferro et al. 2007; Seyahi et al. 2011) CAC significantly correlated with age in both examined groups. In addition, patients from both subgroups with CAC had undergone significantly longer CKD and/or dialysis duration than patients without CAC. An association between CAC and dialysis vintage has already been demonstrated (Rosas et al. 2005). Contrary to Seyahi et al. (2011), we found no association between CAC and transplantation duration.

The differences between renal Tx patients with and without CAC with respect to serum IL-6 and fetuin A levels were not statistically significant. In CKD patients, along with age and CKD duration, both negative acute phase reactants (serum albumin and fetuin A) significantly correlated with CAC. Positive acute phase protein levels tended to be higher in CKD patients with CAC compared to those without CAC. Moreover, after adjusting for age and CKD duration, serum albumin and fetuin A concentrations were significantly lower in the CKD patients with CAC. It is well known that hypo-albuminemia is a marker of inflammation rather than malnutrition. By searching Medline and PubMed databases, Hamirani et al. (2008) found variable association between CAC and inflammation in the general population. Nevertheless, in Tx patients (Mazzaferro et al. 2007) and dialysis patients (Kraśniak et al. 2007; Coen et al. 2006), this relationship was closer in cross-sectional studies and in CAC progression investigations (Mazzaferro et al. 2009; Coen et al. 2010).

Between examined subgroups in $\mathrm{Tx}$ and in CKD patients there were no differences in divalent ion concentrations or for $\mathrm{Ca} \times \mathrm{P}$ product and serum iPTH levels. It has been hypothesized that vascular calcifications in CKD are caused merely by passive deposition of $\mathrm{Ca} \times \mathrm{P}$ products in blood vessels owing to hyper-calcemia and hyper-phosphatemia in conditions of hyper-parathyroidism (Huybers \& Bindels 2007). In dialysis patients, Kranśiak et al. (2007) 
and Coen et al. (2010) found significant correlations between divalent ions and vascular calcification, but other studies did not confirm this (Guérin et al. 2000; Rosas et al. 2005).

During the last ten years, fetuin A, a negative acute phase protein and inhibitor of vascular calcification, has been the focus of vascular calcification by many researchers. Cottone et al. (2010) showed that serum fetuin A was decreased and inflammatory processes increased and linked to endothelial dysfunction even in early stages of CKD. Our results are partially in agreement with some published articles concerning fetuin $\mathrm{A}$ and the CAC score in CKD and Tx patients. The cut-off value of fetuin A to predict the presence of $\mathrm{CAC}$ is equal to $0.437 \mathrm{~g} / \mathrm{L}$ in our study. In Mazzaferro et al. (2009) study, mean values in Tx and dialysis patients were from $0.45 \mathrm{~g} / \mathrm{L}$ to $0.66 \mathrm{~g} / \mathrm{L}$. The optimal cut off values derived from ROC curves was $0.145 \mathrm{~g} / \mathrm{L}$ for fetuin-A, but as a predictor of death in patients with ST-elevation acute myocardial infarction (Lim et al. 2007).

Only a few studies have evaluated the discriminative ability of biomarkers on the extent of CAC in CKD patients. When biological markers of vascular risk and their association with the extent of CAC were considered, fetuin A, malnutrition and inflammation were able to predict the appearance of a significant CAC score. These patients underwent MSCT for suspected coronary artery disease (Uz et al. 2009). In contrast to our study, the AUC for CAC identification was 0.574 for fetuin A. In a study concerning CKD patients, osteoprotegerin had an AUC of 0.79 and neither of calcium phosphate disorder markers had similar ability (Morena et al. 2009).

During the 30 month observation period six of our patients died, four from cardiovascular events. In multivariate analysis age, SAA and CAC score were found to be independent predictors of patient death. Although we only examined a small number of patients, inflammatory markers and severity of CAC together with age were significant predictors of death as already established for inflammatory markers (Simic-Ogrizovic et al. 2009) and CAC score (Coen et al. 2010; Shantouf et al. 2010) in dialysis patients.

Without doubt the present study confirmed the close relationship between age and renal failure and/or dialysis duration before and $\mathrm{CAC}$ after kidney transplantation. One could speculate that multiple established and non-established cardiovascular risk factors at different times during periods of diminished and end stage renal failure contribute to endothelial dysfunction, arterial stiffness and to atherosclerosis and vascular calcifications. At the time of renal transplantation the majority of ESRD patients already have an enormous burden of cardiovascular disease. Hernandez et al. (2005) found pre-existing vascular calcification to be an independent predictor of cardiovascular and all-cause mortality following transplantation. Relapse of pre-existing or appearance new risk factors in the post-transplantation period result, in part, from the atherogenic potential of immunosuppressive drugs (Ojo 2006). Bargnoux et al.
(2009) concluded that successful transplantation prevents de novo uremia-induced calcification in CAC-free patients, stops the progression of pre-existing mild calcification but $\mathrm{CAC}$ progression occurs in recipients with moderate to severe basal CAC.

Successful renal transplantation improves only some negative uremic effects and lowers, but does not halt CAC progression (Mazzaferro et al. 2009). During the posttransplantation period with deteriorating allograft function, recipients again suffer from uremia. Therefore, examination and prevention of risk factors must be started at the beginning of renal failure when the characteristics of favorable and non-favorable aspects of $\mathrm{CAC}$ are more obvious.

In conclusion, the prevalence of CAC in renal transplant patients is relatively high and clearly linked with age and chronic kidney and/or dialysis duration. In CKD patients the prevalence was lower and also correlated with age and CKD duration. Moreover, the negative acute phase markers, serum albumin and fetuin A levels, were associated with CAC. Along with age and serum amyloid A, CAC score was a predictor of patient death. Additional long-term longitudinal studies must incorporate patients at the beginning of chronic renal failure who will be monitored during disease progression, time on dialysis and throughout the kidney transplantation period.

\section{Acknowledgments}

This work was supported by a grant from the Ministry of Science and Technological Development, Republic of Serbia (Project numbers 175089 and 175035).

\section{Conflict of Interest}

All authors have no conflict of interest.

\section{References}

Agatston, A.S., Janowitz, W.R., Hildner, F.J., Zusmer, N.R., Viamonte, M.J. \& Detrano, R. (1990) Quantification of coronary artery calcium using ultra fast computed tomography. $J$. Am. Coll. Cardiol., 15, 827-832.

Bakri, R.S., Afzali, B., Covic, A., Sriskantharan, R., Bharma-Ariza, P., Park, W.H., Sriharan, M., Dalton, N., Wierzbicki, A.S., Crook, M.A. \& Goldsmith, D.J. (2004) Cardiovascular disease in renal allograft recipients is associated with elevated sialic acid or markers of inflammation. Clin. Transplant., 18, 201-208.

Bargnoux, A.S., Dupuy, A.M., Garrigue, V., Jaussent, I., Gahide, G., Badiou, S., Szwarc, I., Deleuze, S., Vernhet, H., Cristol, J.P. \& Mourad, G. (2009) Evolution of coronary artery calcifications following kidney transplantation: relationship with osteoprotegerin levels. Am. J. Transplant., 9, 2571-2579.

Braun, J., Oldendorf, M., Moshage, W., Heidler, R., Zeitler, E. \& Luft, F.C. (1996) Electron beam computed tomography in the elevation of cardiac calcification in chronic dialysis patients. Am. J. Kidney Dis., 27, 394-401.

Coen, G., Manni, M., Angoli, A., Balducci, A., Dessi, M., De Angelis, S., Jankovic, L., Mantella, D., Morosetti, M., Naticchia, A., Nofroni, I., Romagnoli, A., Gallucci, M.T., Tomassini, M., Simonetti, G. \& Splendiani, G. (2006) Cardiac calcifications: fetuin A and other risk factors in hemodialysis patients. ASAIO J., 52, 150-156.

Coen, G., Pierantozzi, A., Spizzichino, D., Sardella, D., Mantella, 
D., Manni, M., Pellegrino, L., Romagnoli, A., Pacifici, R., Zuccaro, P. \& Digiulio, S. (2010) Risk factors of one year increment of coronary calcifications and survival in hemodialysis patients. BMC Nephrol., 11, 1-9.

Cottone, S., Palermo, A., Arsena, R., Riccobene, R., Guarneri, M., Mulè, G., Tornese, F., Altieri, C., Vaccaro, F., Previti, A. \& Cerasola, G. (2010) Relationship of fetuin-A with glomerular filtration rate and endothelial dysfunction in moderate-severe chronic kidney disease. J. Nephrol., 23, 62-69.

Goodman, W.G., Goldin, J., Kuizon, B.D., Yoon, C., Gales, B., Sider, D., Wang, Y., Chung, J., Emerick, A., Greaser, L., Elashoff, R.M. \& Salusky, I.B. (2000) Coronary artery calcification in young adults with end stage renal disease who are undergoing dialysis. N. Engl. J. Med., 342, 1478-1483.

Greenland, P., Bonow, R.O., Brundage, B.H., Budoff, M.J., Eisenberg, M.J., Grundy, S.M., Lauer, M.S., Post, W.S., Raggi, P., Redberg, R.F., Rodgers, G.P., Shaw, L.J., Taylor, A.J. \& Weintraub, W.S. (2007) ACCF/AHA 2007 clinical expert consensus document on coronary artery calcium scoring by computed tomography in global cardiovascular risk assessment and in evaluation of patients with chest pain: a report of the American College of Cardiology Foundation Clinical Expert Consensus Task Force (ACCF/AHA Writing Committee to Update the 2000 Expert Consensus Document on Electron Beam Computed Tomography) developed in collaboration with the Society of Atherosclerosis Imaging and Prevention and the Society of Cardiovascular Computed Tomography. J. Am. Coll. Cardiol., 49, 378-402.

Guérin, A., London, G., Marchais, S. \& Metivier, F. (2000) Arterial stiffening and vascular calcifications in end-stage renal disease. Nephrol. Dial. Transplant., 15, 1014-1021.

Hamirani,Y.S., Pandey, S., Rivera, J., Ndumele, C., Budoff, M.J., Blumenthal, R.S. \& Nasir, K. (2008) Markers of inflammation and coronary artery calcification: a systematic review. Atherosclerosis, 201, 1-7.

Hernandez, D., Rufino, M., Bartolomei, S., González-Rinne, A., Lorenzo, V., Cobo, M. \& Torres, A. (2005) Clinical impact of preexisting vascular calcification on mortality after renal transplantation. Kidney Int., 67, 2015-2020.

Huybers, S. \& Bindels, R.J.M. (2007) Vascular calcification in chronic kidney disease: new developments in drug therapy. Kidney Int., 72, 663-665.

Kestenbaum, B.R., Adeney, K.L., de Boer, I.H., Ix, J.H., Shlipak, M.G. \& Siscovick, D.S. (2009) Incidence and progression of coronary calcification in chronic kidney disease: the MultiEthnic Study of Atherosclerosis. Kidney Int., 76, 991-998.

Ketteler, M., Gross, M.L. \& Ritz, E. (2005) Calcification and cardiovascular problems in renal failure. Kidney Int., 67 (Suppl 94), S120-S127.

Kramer, H., Toto, T., Peshock, R., Cooper, R. \& Victor, R. (2005) Association between chronic kidney disease and coronary artery calcification: the Dallas Heart Study. J. Am. Soc. Nephrol., 16, 507-513.

Kraśniak, A., Drozdz, M., Pasowicz, M., Chmiel, G., Michałek, M., Szumilak, D., Podolec, P., Klimeczek, P., Konieczyńska, M., Wicher-Muniak, E., Tracz, W., Khoa, T.N., Souberbielle, J.C., Drueke, T.B. \& Sulowicz, W. (2007) Factors involved in vascular calcification and atherosclerosis in maintenance haemodialysis patients. Nephrol. Dial. Transplant., 22, 515-521.

Levey, A.S., Coresh, J., Greene, T., Stevens, L.A., Zhang, Y.L., Hendriksen, S., Hendriksen, S., Kusek, J.W. \& Van Lente, F. (2006) Using standardized serum creatinine values in the modification of diet in renal disease study equation for estimating glomerular filtration rate. Ann. Intern. Med., 145, 247-254.
Liefeldt, L. \& Budde, K. (2010) Risk factors for cardiovascular disease in renal transplant recipients and strategies to minimize risk. Transplant. Int., 23, 1191-1204.

Lim, P., Collet, J.P., Moutereau, S., Guigui, N., Mitchell-Heggs, L., Loric, S., Bernard, M., Benhamed, S., Montalescot, G., Randé, J.L. \& Guéret, P. (2007) Fetuin-A Is an Independent Predictor of Death after ST-Elevation Myocardial Infarction. Clin. Chem., 53, 1835-1840.

Martola, M., Barany, P. \& Stenvinkel, P. (2005) Why do dialysis patients develop heart of stone and bone of China? Blood Purif., 23, 203-210.

Mazzaferro, S., Pasquali, M., Pugliese, F., Barresi, G., Carbone, I., Francone, M., Sardella, D. \& Taggim, F. (2007) Serum levels of calcification inhibition proteins and coronary artery calcium score: comparison between transplantation and dialysis. Am. J. Nephrol., 27, 75-83.

Mazzaferro, S., Pasquali, M., Taggi, F., Baldinelli, M., Conte, C., Muci, M.L., Pirozzi, N., Carbone, I., Francone, M. \& Pugliese, F. (2009) Progression of coronary artery calcification in renal transplantation and the role of secondary hyperparathyroidism and inflammation. Clin. J. Am. Soc. Nephrol., 4, 685-690.

Meier-Kriesche, H.U., Schold, J.D., Srinivas, T.R., Reed, A. \& Kaplan, B. (2004) Kidney transplantation halts cardiovascular disease progression in patients with end stage renal disease. Am. J. Transplant., 4, 1662-1668.

Moe, S., O’Neill, K., Resterova, M., Fineberg, N., Persohn, S. \& Meyer, C.A. (2004) Natural history of vascular calcification in dialysis and transplant patients. Nephrol. Dial. Transplant., 19, 2387-2393.

Morena, M., Dupuy, A.M., Jaussent, I., Vernhet, H., Gahide, G., Klouche, K., Bargnoux, A.S., Delcourt, C., Canaud, B. \& Cristol, J.P. (2009) A cut-off value of plasma osteoprotegerin level may predict the presence of coronary artery calcifications in chronic kidney disease patients. Nephrol. Dial. Transplant., 24, 3389-3397.

Ojo, A. (2006) Cardiovascular complication after renal transplantation and their prevention. Transplantation, 82, 603-611.

Rosas, S.E., Mensah, K., Weinstein, R.B., Bellamy, S.L. \& Rader, D.J. (2005) Coronary artery calcification in renal transplant recipients. Am. J. Transplant., 5, 1942-1947.

Schwarz, U., Buzello, M., Ritz, E., Stein, G., Raabe, G., Wiest, G., Mall, G. \& Amann, K. (2000) Morphology of coronary atherosclerotic lesions in patients with end stage renal failure. Nephrol. Dial. Transplant., 15, 218-223.

Seyahi, N., Kahveci, A., Cebi, D., Altiparmak, M.R., Akman, C., Uslu, I., Ataman, R., Tasci, H. \& Serdengecti, K. (2011) Coronary artery calcification and coronary ischaemia in renal transplant recipients. Nephrol. Dial. Transplant., 26, 720-726.

Shantouf, R.S., Budoff, M.J., Ahmadi, N., Ghaffari, A., Flores, F., Gopal, A., Noori, N., Jing, J., Kovesdy, C.P. \& KalantarZadeh, K. (2010) Total and individual coronary artery calcium scores as independent predictors of mortality in hemodialysis patients. Am. J. Nephrol., 31, 419-425.

Simic-Ogrizovic, S., Dopsaj, V., Bogavac-Stanojevic, N., Obradovic, I., Stosovic, M. \& Radovic, M. (2009) Serum amyloid-A rather than C-reactive protein is a better predictor of mortality in hemodialysis patients. Tohoku J. Exp. Med., 219, 121-127.

Swets, J.A. (1988) Measuring the accuracy of diagnostic systems. Science, 240, 1285-1293.

Uz, O., Kardeşoğlu, E., Yiğginer, O., Baş, S., Ipçioğlu, O.M., Ozmen, N., Aparci, M., Cingözbay, B.Y., Işilak, Z. \& Cebeci, B.S. (2009) The relationship between coronary calcification and the metabolic markers of osteopontin, fetuin-A, and visfatin. Turk. Kardiyol. Dern. Ars., 37, 397-402. 